INPLASY

PROTOCOL

To cite: Wu et al. Efficacy and safety of traditional Chinese medicinal enemas for treatment of chronic renal failure: A protocol for systematic review and metaanalysis. Inplasy protocol 202080052. doi: 10.37766/inplasy2020.8.0052

Received: 13 August 2020

Published: 13 August 2020

Corresponding author: Lihua Wu

1025053335@qq.com

Author Affiliation:

Chengdu University of

Traditional Chinese Medicine

Support: Horizontal Scientific Research

Review Stage at time of this submission: Preliminary searches.

Conflicts of interest: None.

\section{Efficacy and safety of traditional Chinese medicinal enemas for treatment of chronic renal failure: A protocol for systematic review and meta-analysis}

Wu, L1; Li, M².

Review question / Objective: Efficacy and safety of traditional Chinese medicinal enemas on chronic renal failure: A protocol for systematic review and meta-analysis.

Condition being studied: Chronic renal failure (CRF) is a clinical syndrome in the later stage of chronic kidney disease. With the development of alternative treatments such as dialysis and transplantation, the curative effect of chronic renal failure has been significantly improved, but it still cannot be used in the early and middle stages and is expensive. In non-dialysis treatment, Chinese medicine is increasingly showing its unique advantages. The traditional Chinese medicine enema method is based on the method of invigorating the kidney and removing turbidity. The enema fluid accelerates the elimination of metabolic waste from the body through the intestine and reduces its damage to important organs in the body. At present, Chinese medicine enema has accumulated rich experience in the treatment of chronic renal failure. In order to objectively evaluate the role of Chinese medicine enema in the treatment of chronic renal failure, this study adopts the evidence-based medical system evaluation method to comprehensively collect the traditional Chinese medicine enema treatment of chronic renal failure. Clinical trial research to evaluate the objective efficacy of TCM enema in the treatment of chronic renal failure, in order to provide reliable evidence for clinical practice.

INPLASY registration number: This protocol was registered with the International Platform of Registered Systematic Review and Meta-Analysis Protocols (INPLASY) on 13 August 2020 and was last updated on 13 August 2020 (registration number INPLASY202080052).

\section{INTRODUCTION}

Review question / Objective: Efficacy and safety of traditional Chinese medicinal enemas on chronic renal failure: A protocol for systematic review and meta-analysis. 
Condition being studied: Chronic renal failure (CRF) is a clinical syndrome in the later stage of chronic kidney disease. With the development of alternative treatments such as dialysis and transplantation, the curative effect of chronic renal failure has been significantly improved, but it still cannot be used in the early and middle stages and is expensive. In non-dialysis treatment, Chinese medicine is increasingly showing its unique advantages. The traditional Chinese medicine enema method is based on the method of invigorating the kidney and removing turbidity. The enema fluid accelerates the elimination of metabolic waste from the body through the intestine and reduces its damage to important organs in the body. At present, Chinese medicine enema has accumulated rich experience in the treatment of chronic renal failure. In order to objectively evaluate the role of Chinese medicine enema in the treatment of chronic renal failure, this study adopts the evidence-based medical system evaluation method to comprehensively collect the traditional Chinese medicine enema treatment of chronic renal failure. Clinical trial research to evaluate the objective efficacy of TCM enema in the treatment of chronic renal failure, in order to provide reliable evidence for clinical practice.

\section{METHODS}

Search strategy: We will comprehensively search the following 9 databases from inception to August 2020: Web of Science, PubMed, EMBASE, Cochrane Central Register of Controlled Trials(CENTRAL), Chinese National Knowledge Infrastructure (CNKI), WANFANE Database, Chinese Scientific and Technological Periodical Database (VIP) and Chinese Biomedical Database (CBM), and the Cochrane Library database.

Participant or population: Patients must be aged at least 18 years, regardless of their sex, race, education, or economic status. *Patients with CRF, and the diagnosis of CRF should refer to the standard diagnostic criteria including (1) Endogenous creatinine clearance rate
(CCR) 133umol/L. (3) Has a history of chronic kidney disease or systemic disease involving the kidney.

Intervention: The purpose of the study is on clinical trials of traditional Chinese medicinal enemas for CRF. The intervention group can combine other conventional treatments; while the control group will only include only other conventional treatments.

Comparator: Conventional CRF treatments and placebo or conventional treatments only.

Study designs to be included: The only randomized controlled trial will be included. Non-RCTs and uncontrolled clinical trials will be excluded.

Eligibility criteria: Only RCTs (except QuasiRCTs and cluster RCTs) will be included. Animal mechanism studies and non-randomized clinical trials will be excluded. For the included trials, the investigators need to precisely report Chinese herb medicine treatment details and parameters, diagnostic criteria, and efficacy evaluation they based on. There is no unified requirement on the blinding and language of the findings. No limitation to whether it is published or not. Language is limited to Chinese and English.

Information sources: We will comprehensively search the following 9 databases from inception to August 2020: Web of Science, PubMed, EMBASE, Cochrane Central Register of Controlled Trials(CENTRAL), Chinese National Knowledge Infrastructure (CNKI), WANFANE Database, Chinese Scientific and Technological Periodical Database (VIP) and Chinese Biomedical Database (CBM), and the Cochrane Library database. Ambiguous literature will be manually searched to avoid missing eligible trials. Ongoing registered clinical trials will be searched on the websites of the Chinese clinical trial registry (http:// www.chictr.org.cn/) and international clinical trial registry (http:// clinicaltrials.gov/). Additional trials will be 
searched by reviewing the reference lists of the retrieved articles, conference proceedings, and gray literature.

Main outcome(s): The primary outcomes include blood urea nitrogen, uric acid, Ccr, Scr.

Additional outcome(s): The secondary outcomes include clinical efficacy and adverse events. The clinical efficacy refers to the guiding principles for clinical research of new Chinese medicines and is determined according to the degree of improvement of the symptoms of the patient before and after treatment: markedly effective: clinical symptoms are reduced or disappeared, Ccr increased by $\geq \mathbf{2 0} \%$ or Scr decreased by $\mathbf{2} \mathbf{2 0} \%$. Effective: Clinical symptoms are reduced or disappeared, Ccr increased by $\geq 10 \%$ or Scr decreased by $\geq 10 \%$. Ineffective: Clinical symptoms are not significantly relieved, Ccr increase $\leq 10 \%$ or Scr decrease $\leq 10 \%$.

Quality assessment / Risk of bias analysis: Quality assessment will be performed using the tool for "risk of bias" from the Cochrane Handbook for Systematic Reviews of Interventions. Random sequence generation, allocation concealment, the use of blindness, the integrity of the outcome data, selective outcome reporting, and other risks of bias will be assessed by 2 reviewers (TJ and LhW). According to the above 6 items, each study will be assessed as "high risk," "low risk," or "unclear risk." For unclear items in the study, contact the corresponding author for details. Any disagreement will be resolved by discussion with a third reviewer (M-qL).

Strategy of data synthesis: Binary outcomes will be summarized using risk ratio (RR) with a $95 \%$ confidence interval (CI) for relative effect. When the heterogeneity test indicated the existence of heterogeneity between studies $(P \leq .01$, $12 \geq 50 \%$ ), the random-effect model was used for combined analysis, or subgroup analysis or sensitivity analysis was conducted according to the heterogeneity source. When the heterogeneity test indicated that there was no heterogeneity between the groups ( $P>01,12<50 \%$ ), the fixed-effect model was used for combined analysis. If quantitative synthesis is not appropriate, qualitative analysis will be carried out.

Subgroup analysis: Subgroup analyses will be performed to explain heterogeneity if possible. Factors such as different CHM dosage forms, intervention forms, measurements of results will be considered. If a meta-analysis cannot be performed, we will conduct a descriptive analysis instead.

Sensibility analysis: When the heterogeneity is significantly different from the methodological quality of the included studies, the stability of the results can be assessed by sensitivity analysis. The effect of methodological quality, sample size, and missing data will be assessed. In addition, the analysis will be repeated after excluding low-methodological quality studies. If quantitative synthesis is not appropriate, a comprehensive narrative will be provided through the text and tabular information to summarize and explain the characteristics and findings of the literature, and to explore the relationship within or between the literature.

Country(ies) involved: China.

Keywords: chronic renal failure; traditional Chinese medicinal enemas; systematic review.

Contributions of each author:

Author 1 - Lihua, Wu.

Author 2 - Mingquan Li. 